\title{
Detecting Regional Abnormal Cardiac Contraction in Short-Axis MR Images Using Independent Component Analysis ${ }^{\star}$
}

\author{
A. Suinesiaputra ${ }^{1}$, M. Üzümcü ${ }^{1}$, A.F. Frangi ${ }^{2, \star \star}{ }^{, \text {T.A.M. Kaandorp }}{ }^{1}$, \\ J.H.C. Reiber ${ }^{1}$, and B.P.F. Lelieveldt ${ }^{1}$ \\ 1 Division of Image Processing, Department of Radiology, \\ Leiden University Medical Center, Leiden, The Netherlands \\ 2 Computer Vision Group, Aragon Institute of Engineering, \\ University of Zaragoza, Zaragoza, Spain
}

\begin{abstract}
Regional myocardial motion analysis is used in clinical routine to inspect cardiac contraction in myocardial diseases such as infarction or hypertrophy. Physicians/radiologists can recognize abnormal cardiac motion because they have knowledge about normal heart contraction. This paper explores the potential of Independent Component Analysis (ICA) to extract local myocardial contractility patterns and to use them for the automatic detection of regional abnormalities. A qualitative evaluation was performed using 42 healthy volunteers to train the ICA model and 6 infarct patients to test the detection and localization. This experiment shows that the evaluation results correlate very well to the clinical gold standard: delayed-enhancement MR images.
\end{abstract}

\section{Introduction}

Identification of reversible myocardial ischemic injury is a crucial assessment before coronary revascularization. Myocardial infarction is characterized by the presence of hypo-kinetic regions. MRI images have been used widely to diagnose myocardial infarction, especially recently with the delayed-enhancement MRI [1].

The effect of coronary artery occlusion is an abnormal myocardial contraction, particularly in the infarcted regions. Figure 1 shows two examples of MRI images from a healthy volunteer and an infarct patient, both at end-systole. Note that the inferior region (indicated by a white arrow) of the infarct heart does not contract, and has a reduced wall thickness.

The goal of this work is to automate the detection of abnormal cardiac motion from short-axis MRI images. This is achieved by deriving a statistical model of

* This work is supported by the Dutch Science Foundation (NWO), under an innovational research incentive grant, vernieuwingsimpuls 2001.

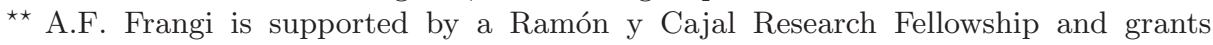
TIC2002-04495-CO2 and ISCIII G03/185 from the Spanish Ministries of Science \& Technology, and Health, respectively. 


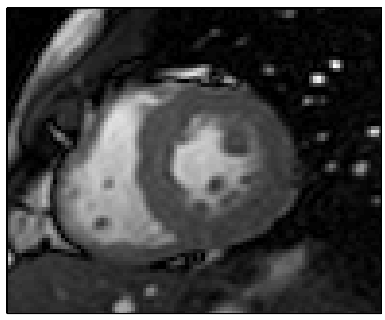

(a) healthy volunteer

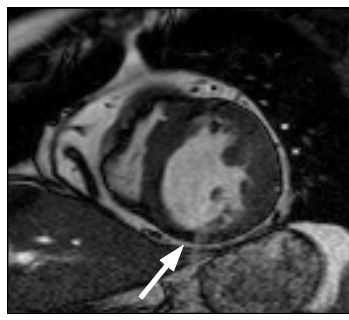

(b) infarct patient

Fig. 1. MRI images of a healthy volunteer and an infarct patient at end-systole. The white arrow points to the infarcted tissue.

normal heart contraction and its local contractility patterns. In this paper, ICA is used to model the normal heart contraction and to detect and localize regions of the abnormal contraction in a patient. The contributions of this paper are threefold:

- We propose a geometry-based sorting method of independent components, providing an intuitive anatomical interpretation of the ICA modes.

- We demonstrate the potential of ICA in cardiac shape modeling to detect local contraction abnormalities.

- We present a qualitative evaluation of the detection and localization of myocardial infarctions. Results are compared with the corresponding "goldstandard" delayed-enhancement MRI images.

Section 2 describes shape modeling with ICA, the new sorting method for independent components and the method to detect local abnormalities. In Section 3 qualitative evaluation results are presented, followed by a discussion in Section 4

\section{Methodology}

\subsection{ICA Modeling of the Normal Cardiac Contraction}

ICA is originally used for finding source signals from a mixture of unknown signals without prior knowledge other than the number of sources. In machine learning, ICA has been applied for feature extraction [2] and face recognition [3]. ICA can be applied to statistical shape modeling to extract independent components of the shape variation [4].

ICA is a linear generative model, where every training shape can be approximated by a linear combination of its components. Let $\boldsymbol{x}=\left(x_{1}, y_{1}, \ldots, x_{m}, y_{m}\right)^{T}$ be a shape vector, consisting of $m$ pairs of $(x, y)$ coordinates of landmark points. The linear generative model is formulated as follows:

$$
\boldsymbol{x} \approx \overline{\boldsymbol{x}}+\boldsymbol{\Phi} \boldsymbol{b} .
$$


The matrix $\boldsymbol{\Phi} \in \mathbb{R}^{2 m \times p}$ defines the independent components (ICs) and $\boldsymbol{b} \in \mathbb{R}^{p}$ is the weight coefficient vector. The mean shape, $\overline{\boldsymbol{x}}$, is defined by

$$
\overline{\boldsymbol{x}}=\frac{1}{n} \sum_{i=1}^{n} \boldsymbol{x}_{i} .
$$

where $n$ is the number of shapes and $p$ is the number of retained components.

The goal of ICA is to find a matrix, $\boldsymbol{\Psi}$, such that

$$
\boldsymbol{b}=\boldsymbol{\Psi}(\boldsymbol{x}-\overline{\boldsymbol{x}})
$$

with a constraint that columns of $\boldsymbol{\Psi}$ correspond to statistically independent directions. Thus the independent components are given by $\boldsymbol{\Phi}=\boldsymbol{\Psi}^{-1}$. The matrix $\boldsymbol{\Psi}$ is estimated by an optimisation algorithm (see 5 for survey of ICA).

Some pre-processing steps are necessarily performed before the ICA computation. The training shapes must be aligned, such that all shapes are invariant under Euclidean similarity transformations (rotation, translation and scaling). Procrustes analysis [6] is used for the shape alignment. Point correspondence between shapes is usually obtained by taking landmark points with the same anatomical interpretation. The resulting training shapes are zero mean, unit variance and all points are registered between shapes.

In this application, the observed data are left ventricular (LV) myocardial contours from short-axis cardiac MRI images at end-diastole (ED) and endsystole (ES) phases. To model the contractility pattern, contours for each subject are combined serially into one shape vector in the following order: endocardium contour at ED, epicardium contour at ED, endocardium contour at ES and epicardium contour at ES.

Figure 2(a) shows one example of an ICA derived shape variation mode. For comparison, the first mode of shape variation with PCA from the same data is shown in Fig. 2(b). ICA modes have a general shape of a local "bump", whereas the remainder of the shape is unaffected. This is an important property of ICA, which can be used to detect local shape anomalies. In contrast, PCA modes give global shape variations, distributed over the entire contour. A comparison study of ICA and PCA in cardiac shape modeling is given in [4].

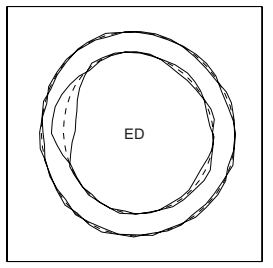

(a) ICA mode
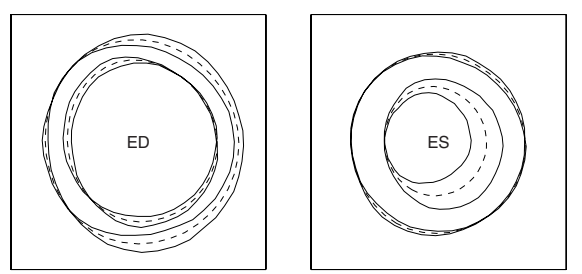

(b) PCA mode

Fig. 2. Examples of modes from ICA and PCA models. The mean shape is drawn with a dashed line. The mode is drawn with a solid line. The shape variations are drawn with $\pm 3 \sigma$ of the weight matrix distribution from the mean shape. 


\subsection{Geometry-Based Sorting of Independent Components}

In our study, ICA is used to detect regional abnormalities, which are extracted from local shape variations. It would give a benefit in this case, if the position of each IC can be determined. Thus ICs are ordered based on their positions along the whole contour, giving an anatomically meaningful interpretation.

Let $\hat{\boldsymbol{x}}_{i}$ be a shape vector from the $i$-th component

$$
\hat{\boldsymbol{x}}_{i}=\overline{\boldsymbol{x}}+\boldsymbol{\Phi} \boldsymbol{a}
$$

where $1 \leq i \leq p, a_{i}=1$ and $a_{j}=0$ for $j \neq i$. A displacement vector $\boldsymbol{d}_{i} \in \mathbb{R}^{m}$ is defined as the distance of each element of $\hat{\boldsymbol{x}}_{i}$ to the mean shape

$$
\boldsymbol{d}_{i}^{(j)}=\sqrt{\sum_{k=2 j-1}^{2 j}\left(\hat{\boldsymbol{x}}_{i}^{(k)}-\overline{\boldsymbol{x}}^{(k)}\right)^{2}} \quad \text { where } j=1,2, \ldots, m .
$$

To determine the position of an independent component, a normalized circular cross-correlation is performed to each contour from the displacement vector with a bank of Gaussian filters. The parameter of the Gaussian filter giving the maximum response are stored for each component. The center of this filter defines the position of the component. Figure 3(a) shows an example of the cross-correlation response from a component.

There is an advantage of this sorting mechanism, that noise components can be detected. Noise components have a global wrinkled shape variation along the whole contour, which correlates best with the widest Gaussian filter. Figure 3(b) shows an example of the cross-correlation response for a noise component. Noise components are thus easily eliminated.

Figure 4 shows an example of the first four ICA modes after the geometrybased sorting mechanism. Note that the local shape variations are orderded clockwise.

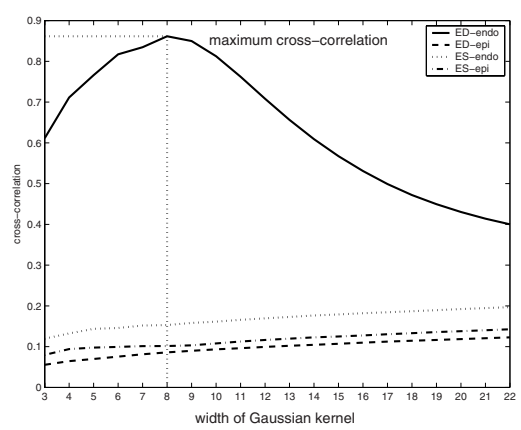

(a) A non-noise IC.

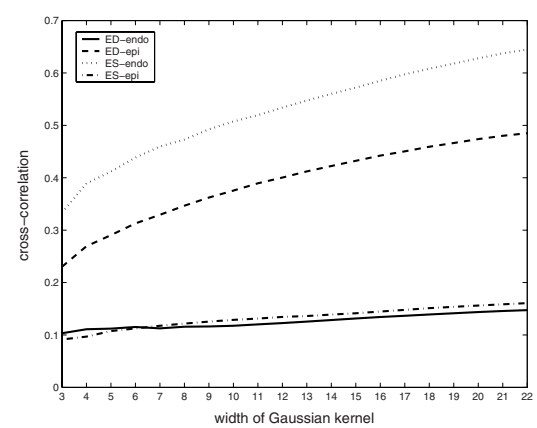

(b) A noise IC.

Fig. 3. Cross-correlation responses from two ICs. 


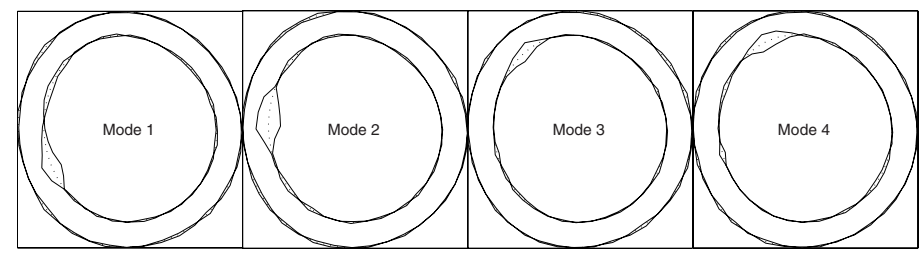

Fig. 4. The first four sorted ICA modes.

\subsection{Determining the Number of Independent Components}

One important parameter to determine is the number of independent components to estimate during the computation of ICA. Predicting this number with PCA may not always be a good idea, because PCA has a risk to eliminate "weak" ICs in the reduced data [7. In shape modeling, this parameter affects appearance of the shape variations. As the number of computed ICs increases, the components represent more localized shape variations. If this parameter is too small, then the component gives global shape variation, much like PCA modes.

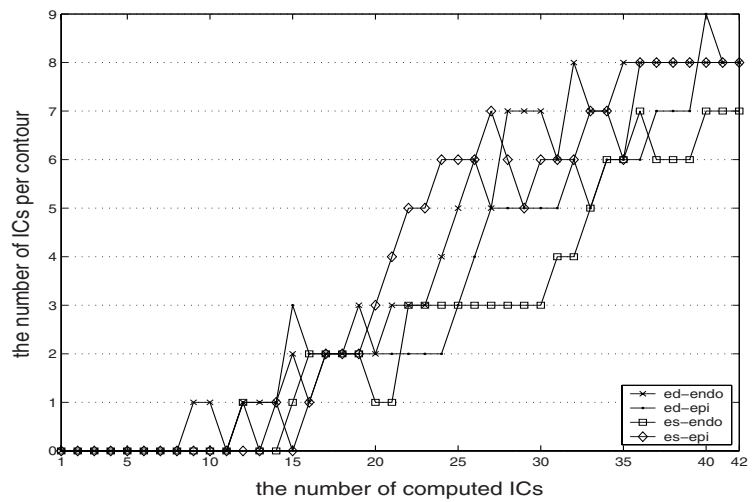

Fig. 5. A plot of the number of ICs per contour as a function of the number of computed ICs.

The determination of the optimal number of computed ICs is therefore taskspecific. In this application to detect local abnormalities, we need sufficient regional segments. Too few segments will give an inaccurate localization. More segments will improve the detection resolution, but this is constrained by the computation time and the number of available shapes to avoid overlearning [8].

Figure 5 shows the number of segments as a function of the number of computed ICs from 42 shapes of normal hearts. From this, we took 40 as the number of computed ICs in our experiment, as it gives enough segments per contour. 


\subsection{Detection of Abnormal Contractility Patterns}

Let $\boldsymbol{y} \in \mathbb{R}^{2 m}$ be a shape vector, fitted onto the mean shape of the model using the Procrustes fit [ $[\underline{6}$. The weight vector of the sample $\boldsymbol{y}$ is given by

$$
\boldsymbol{b}_{\mathrm{y}}=\boldsymbol{\Phi}^{-1}(\boldsymbol{y}-\overline{\boldsymbol{x}})
$$

which represents the parameters approximating the patient shape. Patient anomalies are estimated by elements in the weight vector that lie outside the distribution of parameters of the ICA model.

We have made a statistical test to test the gaussianity for the distribution of coefficient values for each component. We can assume that all components have normal distribution.

We define the anomaly at the i-th component $q_{\mathrm{y}}^{(i)}$ as a value that falls beyond $\pm 3 \sigma_{i}(99.7 \%)$, to make sure that the anomaly is an outlier. Thus the anomaly vector $q_{\mathrm{y}}$ is defined by taking the outlier components, normalized by their standard deviation. Each element of $q_{\mathrm{y}}$ is defined by

$$
q_{\mathrm{y}}^{(i)}=\left\{\begin{array}{l}
0 \quad \text { if }-3 \sigma_{i} \leq b_{\mathrm{y}}^{(i)} \leq 3 \sigma_{i} \\
\frac{b_{\mathrm{y}}^{(i)}}{\sigma_{i}} \text { otherwise }
\end{array} \quad \text { for } i=1, \ldots, p\right.
$$

The anomaly vector (7) is mapped to a shape vector to facilitate a more intuitive regional interpretation. From the sorted ICs, the corresponding Gaussian filters giving the maximum responses for each IC are known. These Gaussian filters are generated to model the local bumps, resulting in a mixture of Gaussian functions. The regional sum of the Gaussian mixture gives a shape vector that indicates regional abnormal heart contraction of a patient.

\section{$3 \quad$ Experimental Results}

An ICA model was constructed from 42 healthy volunteers. The mid-ventricular level from short-axis MRI was taken from each subject. Contours were drawn manually and resampled to 40 landmarks defined by equi-angular sampling, starting from the intersection between left and right ventricle. The calculation of ICA was performed using the JADE algorithm 9], implemented in Matlab. The optimal number of computed ICs with minimum of 7 segments per contour is 40 (see Fig. 5).

To evaluate the infarct detection and localization of our method, MRI data of 6 patients with all necrotic infarcts were investigated. Mid-ventricular shortaxis (SA) MRI images and the corresponding delayed-enhancement (DE) MRI images with the same orientation and the distance only $<1 \mathrm{~mm}$ were acquired. Regional abnormal contraction was compared visually with the corresponding DE-MRI. The myocardial infarct regions in the DE-MRI are demonstrated by signal hyperenhancement, corresponding to myocardial necrosis [1].

Six representative evaluation results are presented in Fig. 6. The anomaly vectors of patients were projected to the corresponding myocardial regions. The 


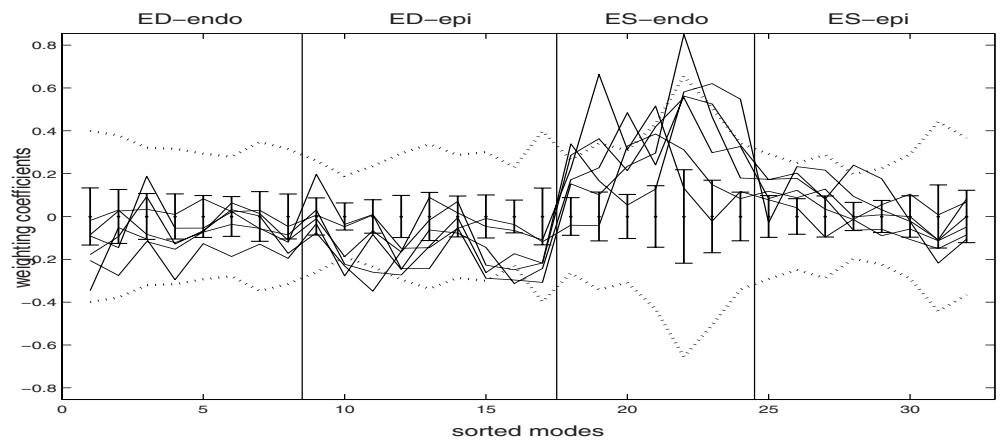

(a) Weight vectors (solid lines) of patients with the distribution of the ICA model (error bars). The dotted lines are the boundary of the normal contraction $\left( \pm 3 \sigma_{i}\right.$ for $\left.i=1, \ldots, p\right)$.

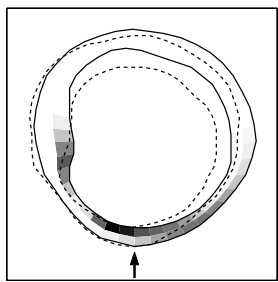

(b) Infarction in the inferior wall.
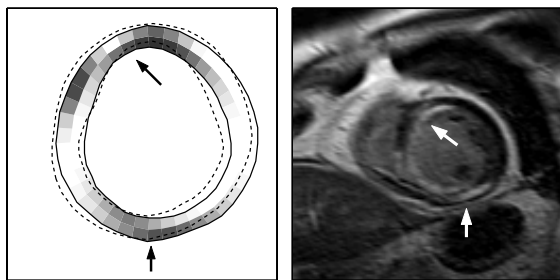

(d) Multiple infarctions.

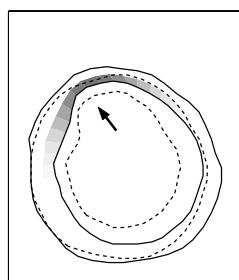

(f) Infarction in the septal wall.
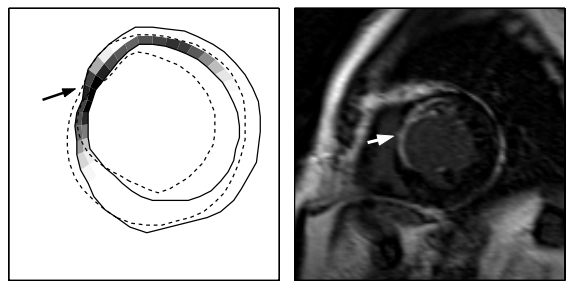

(c) Infarction in the septal wall.
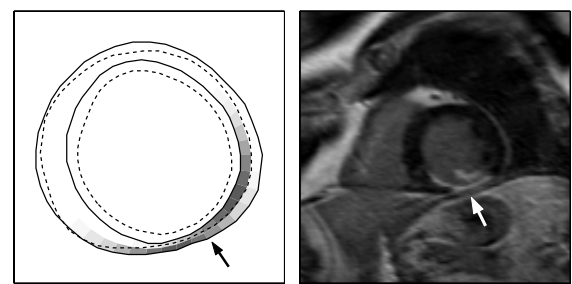

(e) Infarction in the inferior wall.
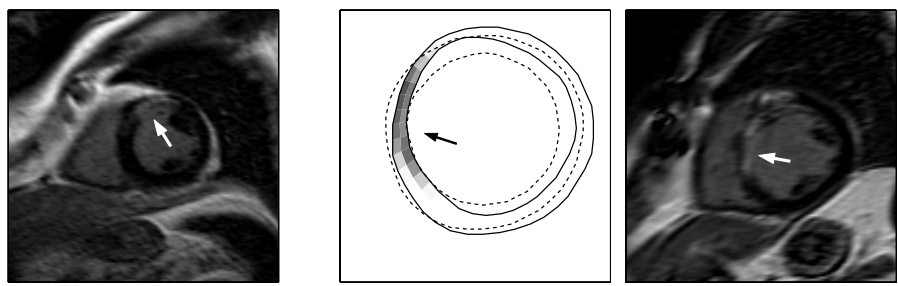

(g) Infarction in the septal wall.

Fig. 6. Qualitative evaluation results. The top row figure shows projection of 6 patients to the ICA model of normal heart. Their abnormalities are shown below in the myocardial regions $(\mathrm{ED}=$ solid, $\mathrm{ES}=$ dashed). Dark areas have high abnormality value, whereas white areas are normal. The corresponding DE-MRI images are shown at the right side. 
contraction patterns are also shown in the plot of ED contours (solid line) and ES contours (dashed line). It is clearly seen from Fig. 6, that the dark areas have a reduced contraction. The corresponding DE-MRI are given in the right side where the infarction regions are depicted by hyperintensity regions.

\section{Discussion}

This study shows the potential of ICA as an analysis tool for extracting local shape deformation. Using ICA to train a model of normal cardiac contraction, both global and regional motions are captured. To this end, we can automatically distinguish between abnormal and healthy cardiac motion.

An intuitive anatomical interpretation of the normal contraction model is achieved by ordering the ICs of the model geometrically along the whole contour. From this, anatomical shape information can be inferred, providing a method to localize the motion abnormalities.

In the qualitative comparison for 6 patients, the hypo-kinetic regions show an excellent correspondence to the hyperintensity regions of the "gold standard" DE-MRI. This demonstrates that the ICA-based infarct detection and localization from short-axis MRI images is a promising technique for computer aided infarct localization.

\section{References}

[1] Kim, R., Wu, E., Rafael, A., et. al.: The Use of Contrast-Enhanced Magnetic Resonance Imaging to Identify Reversible Myocardial Dysfunction. New England Journal of Medicine 343 (2000) 1445-1454

[2] Hoyer, P.O., Hyvärinen, A.: Independent Component Analysis Applied to Feature Extraction from Colour and Stereo Images. Network: Computation in Neural System 11 (2000) 191-210

[3] Bartlett, M.S., Movellan, J.R., Sejnowski, T.J.: Face Recognition by Independent Component Analysis. IEEE Trans. on Neural Networks 13 (2002) 1450-1464

[4] Üzümcü, M., Frangi, A.F., Reiber, J.H., Lelieveldt, B.P.: Independent Component Analysis in Statistical Shape Models. In Sonka, M., Fitzpatrick, J.M., eds.: Proc. of SPIE. Volume 5032. (2003) 375-383

[5] Hyvärinen, A.: Survey on Independent Component Analysis. Neural Computing Surveys 2 (1999) 94-128

[6] Dryden, I.L., Mardia, K.V.: Statistical Shape Analysis. John Wiley \& Sons (2002)

[7] Nadal, J.P., Korutcheva, E., Aires, F.: Blind Source Separation in the Presence of Weak Sources. Neural Network 13 (2000) 589-596

[8] Hyvärinen, A., J.Särelä, Vígario, R.: Bumps and Spikes: Artifacts Generated by Independent Component Analysis with Insufficient Sample Size. In: Proc. Int. Workshop on ICA and BSS. (1999) 425-249

[9] Cardoso, J., Souloumiac, A.: Blind Beamforming for Non Gaussian Signals. IEEE Proceedings-F 140 (1993) 362-370 